\title{
L'influence des représentations sociales dans le développement professionnel des futurs enseignants : le cas de l'évaluation des apprentissages
}

Nicole Monney, Université du Québec à Chicoutimi nicole1.monney@uqac.ca,

Monique L'Hostie, Université du Québec à Chicoutimi Monique Lhostie@uqac.ca

Sylvie Fontaine, Université du Québec en Outaouais Sylvie.Fontaine@uqo.ca 


\section{REVUE HYBRIDE DE L’ÉDUCATION}

\section{Résumé}

Cet article présente les résultats d'une recherche visant à dégager les représentations sociales (RS) de l'évaluation des apprentissages chez quatre stagiaires finissantes en enseignement primaire. Les chercheurs ont opté pour la méthode de l'entretien d'autoconfrontation pour cerner l'interaction entre les RS et les savoirs universitaires. L'analyse réalisée à partir de la théorie des RS et des processus d'objectivation et d'ancrage met de l'avant une résistance au changement dans les pratiques évaluatives. La conclusion soulève des pistes de réflexion pour revoir les rôles des formateurs, la place du stage et le statut des savoirs issus de la recherche.

Mots-clés: Représentations sociales; évaluation des apprentissages; développement professionnel; formation initiale; formation pratique. 


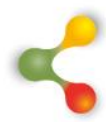

\section{REVUE HYBRIDE DE L'ÉDUCATION}

\section{Le développement professionnel et les difficultés d'arrimer savoirs théoriques et expérientiels}

Depuis 2001, au Québec, la formation à l'enseignement adopte la perspective de la professionnalisation (ministère de l'Éducation du Québec [MEQ], 2001) : « Cette perspective exprime l'idée de développement et de construction de compétences nécessaires à l'exercice d'une profession » (p.17). Selon Uwamariya et Mukamurera (2005), la perspective de professionnalisation propose deux orientations: le développement professionnel par le processus d'apprentissage et le développement professionnel par la recherche ou la réflexion. Le développement professionnel par le processus d'apprentissage réfère à une vision constructiviste qui considère l'enseignant comme un apprenant qui construit et déconstruit ses savoirs tout au long de sa carrière (Uwamariya et Mukamurera, 2005). Le développement professionnel par la recherche ou par la réflexion rejoint les travaux de Schön (1987) et de St-Arnaud (1992) qui abordent la réflexion dans l'action et la réflexion sur l'action. À l'instar de Vanhulle (2008), nous reconnaissons que le développement professionnel se définit au-delà de la perspective d'apprentissage. Le processus de réflexion permet une intégration de savoirs investis de sens qui n'apportent pas uniquement de nouveaux savoirs à l'apprenant, mais qui transforment son fonctionnement psychique (Buysse et Vanhulle, 2009). Le développement professionnel ne peut être effectif sans la réflexion sur son action.

Par souci de cohérence avec cette orientation, la formation à l'enseignement au Québec cible le développement de 12 compétences professionnelles. Le choix d'opter pour le développement des compétences influence la structure de la formation. Par exemple, le concept de compétence suppose un développement en contexte professionnel réel et sur une longue durée. Ainsi, la formation dure quatre années et propose une alternance entre les cours universitaires et les stages, le but ultime étant de favoriser l'ancrage des savoirs universitaires dans les milieux pratiques. La compétence amène aussi l'idée d'un continuum dans le temps, d'un projet, ou encore d'une finalité sans fin. Ainsi, dès le début de la formation initiale, les enseignants s'engagent dans une démarche de développement professionnel qui se poursuivra tout au long de leur parcours. Les concepteurs du programme de formation à l'enseignement ont d'ailleurs souligné l'importance de cet engagement en faisant une compétence spécifique dans le référentiel des 12 compétences (MEQ, 2001), à savoir la compétence à " s'engager dans une démarche individuelle et collective de développement professionnel » (p. 125-129). Cette compétence réfère précisément au processus de réflexion tel que proposé par Vanhulle (2008).

Cependant, structurer la formation autour de compétences et viser le développement professionnel des futurs enseignants par le biais de la réflexion ne semble pas suffisant. Les futurs enseignants ont beaucoup de 


\section{6}

\section{REVUE HYBRIDE DE L'ÉDUCATION}

difficulté à passer des formes décontextualisées (savoirs théoriques, didactiques, pédagogiques et expérientiels) aux formes contextualisées (pratiques en classe) et inversement (Desjardins, 2013). Le cas de la compétence à évaluer, compétence 5 du référentiel (MEQ, 2001), en est un exemple. Les futurs enseignants semblent s'appuyer sur leurs propres expériences en tant qu'élèves pour mettre en place des pratiques évaluatives (Skamp, 2001), et ce, dans l'idée que l'évaluation a un rôle de sanction plutôt que de soutien à l'apprentissage comme c'est le cas de l'évaluation formative (Monney, 2014). Dans un contexte où les recherches sur l'évaluation tendent à souligner l'importance de l'évaluation formative sur la réussite éducative (de Champlain, 2011; Dirksen, 2013 ; Stiggins, 2009), ce constat est questionnable. Par ailleurs, outre le fait que l'évaluation formative n'est pas une visée dans les pratiques des futurs enseignants, ils se disent également peu à l'aise dans leur rôle d'évaluateur des apprentissages de leurs élèves (Bidjang, Gauthier, Mellouki et Desbiens, 2005 ; Fontaine, Kane, Duquette et Savoie-Zajc, 2011 ; Mertler, 2009).

Les futurs enseignants suivent au moins un cours spécifique portant sur l'évaluation des apprentissages et plusieurs cours de didactique qui introduisent les concepts d'évaluation formative. Ils réalisent également des stages durant lesquels ils ont la possibilité d'objectiver les savoirs didactiques, théoriques ainsi que pédagogiques et de les subjectiver en savoirs pratiques (Vanhulle, 2008). Il y a donc lieu de se questionner sur les raisons qui amènent ces difficultés chez les futurs enseignants à intégrer les savoirs issus des cours universitaires aux pratiques en classe, et ce, par rapport à la compétence à évaluer et aux savoirs à mobiliser pour le développement de celle-ci.

\section{Cadre théorique}

\section{L'évaluation des apprentissages}

Lorsqu'on mentionne le concept d'évaluation des apprentissages, la première image qui nous vient à l'esprit est souvent celle de l'examen écrit comprenant plusieurs questions à points. Cet examen peut prendre la forme d'un questionnaire à choix multiples ou encore d'un ensemble de questions à développement. Des souvenirs négatifs peuvent également être associés à ce type d'examen tels qu'une mauvaise note, un échec, des sueurs froides durant l'épreuve, des pertes de mémoire, de l'anxiété, etc. Or, depuis 30 ans, les programmes d'enseignement au Québec et ailleurs dans le monde font la promotion d'une évaluation dite formative (Legendre, 2004 ; Morrissette, 2009 ; Scallon, 1986) visant à soutenir l'élève pendant l'apprentissage. Cette évaluation est intégrée à la dynamique des apprentissages de l'élève et fournit une information diagnostique pour guider l'enseignement et l'apprentissage (Laurier, 2014). Dans le monde anglo-saxon, il existe un terme spécifique pour ce type d'évaluation : «formative assessment ». Cela permet de bien distinguer la 


\section{6}

\section{REVUE HYBRIDE DE L'ÉDUCATION}

démarche d'accompagnement de l'élève durant la séquence d'enseignement de la démarche évaluative axée sur la sanction des apprentissages en fin de séquence (Dunn et Mulvenon, 2009). L'évaluation formative se réalise ainsi dans une démarche qui favorise un retour à l'élève sur ses apprentissages et sur les processus utilisés (Hattie et Timperley, 2007) dans le but de réguler les apprentissages, les processus ou l'enseignement (Allal et Mottier Lopez, 2007a). Toute activité qui vise à fournir une rétroaction à l'élève au sujet de ses apprentissages devient ainsi une occasion d'évaluation formative (Black et Wiliam, 1998). Par exemple, l'enseignant peut partager les buts d'apprentissage avec les élèves, apporter des propositions d'amélioration, offrir des outils ou des stratégies d'autoévaluation ainsi qu'aider les élèves à réfléchir aux apprentissages qu'ils sont en train de faire en les questionnant régulièrement (Stiggins, 2009). Ainsi, il ne s'agit pas uniquement de faire passer un examen écrit aux élèves, mais bien d'identifier tout au long des interactions en classe les difficultés et les forces des élèves et de leur en faire part pour leur permettre d'atteindre les objectifs d'apprentissage visés par le programme.

La problématique met de l'avant l'importance du processus de réflexion pour favoriser le développement professionnel et, dans le cas de cet article, le développement de la compétence à évaluer. Or, les futurs enseignants ont beaucoup de difficulté à passer des formes décontextualisées (savoirs théoriques, didactiques, pédagogiques et expérientiels) aux formes contextualisées (pratiques en classe) et inversement (Desjardins, 2013). Une hypothèse soumise dans cet article est le fait que les futurs enseignants semblent s'appuyer sur leurs propres expériences en tant qu'élève pour mettre en place des pratiques évaluatives (Skamp, 2001). L'évaluation des apprentissages étant avant tout un objet social puisque toute personne a déjà été évaluée à un moment, le deuxième concept central à cet article est la représentation sociale (RS).

\section{La représentation sociale}

Une RS est une forme de connaissance, socialement élaborée et partagée orientant l'action et concourant à la construction d'une réalité commune à un ensemble social (Jodelet, 1989). Elle constitue un univers d'opinions (Moscovici, 1961), d'informations, de croyances et d'attitudes se référant à un objet ou à une situation (Abric, 1994). Selon Buysse et Vanhulle (2009), " tout apprentissage s'inscrit à l'intérieur d'une forme culturelle d'action et de réflexion historiquement marquée, située dans des pratiques sociales, médiatisée par des langages, des interactions, des artéfacts » (p.228). Ainsi, lorsqu'un futur enseignant entreprend une démarche réflexive sur ses apprentissages, cette démarche se réalise nécessairement dans un contexte culturel rattaché à la profession et dans un contexte où différentes RS circulent. Pour le futur enseignant, autant les savoirs théoriques, didactiques, pédagogiques que ceux issus de 


\section{6}

\section{REVUE HYBRIDE DE L'ÉDUCATION}

l'expérience font partie de la RS du métier. Les différents éléments constituants la RS du métier se sont ancrés au fil du parcours en tant qu'élève ou étudiant (Doyle, 1997; Karp et Woods, 2008; Parker et Brindley, 2008). Cet ancrage des RS suit un processus en deux temps : le processus d'objectivation et le processus d'ancrage.

\section{Processus d'objectivation et d'ancrage de la RS}

Durant le processus d'objectivation, l'individu déforme les savoirs enseignés en privilégiant certaines informations au détriment d'autres éléments et en les dissociant de leur contexte initial beaucoup trop complexe pour l'individu (Bonardi et Roussiau, 1999). En d'autres termes, un futur enseignant, lors d'un cours universitaire, reçoit des informations en lien avec un concept propre au contexte professionnel (évaluation des apprentissages, gestion de la classe, etc.). II va transformer cet objet abstrait (le concept) en une image significative pour lui. Par exemple, il lie ce nouveau concept à une situation connue comme élève. II décide inconsciemment de rejeter certains aspects de ce concept et d'en garder d'autres qui s'ajustent mieux à sa représentation du concept présenté. Ensuite, dans un deuxième mouvement, ces savoirs transformés passent par un processus d'ancrage. Ce deuxième mouvement permet de se réapproprier les savoirs dans un contexte social. II s'agit du deuxième mécanisme, l'ancrage, qui consiste à enraciner cette nouvelle image dans la RS et à faire un lien avec l'espace social dans lequel vit l'individu. Le processus de réflexion proposé par la théorie du praticien réflexif (Schön, 1987) pourrait aider le processus d'ancrage de la RS où le praticien réfléchit sur l'action à partir de ses savoirs.

La question se pose donc à savoir quelle est l'influence des RS sur les pratiques évaluatives des finissants en enseignement. La première partie de l'étude dont il est question ici consistait à décrire la structure de la RS de l'évaluation des apprentissages chez une cohorte de finissants à partir de la technique d'évocation hiérarchisée (Vergès, 1992). Les résultats ont montré que la RS de l'évaluation des apprentissages intègre le concept d'évaluation formative dans une visée de régulation des apprentissages (Monney et Fontaine, 2016). Or, qu'en est-il dans l'action ? À l'instar de Jorro (2014), nous pensons que le milieu transforme également l'acteur. C'est d'ailleurs la raison pour laquelle la deuxième phase de l'étude a été réalisée en contexte de stage et visait à mettre en évidence l'influence des RS sur le développement de leurs pratiques évaluatives.

\section{Méthodologie}

Selon Pajares (1992), l'étude des RS demande de considérer trois dimensions: le discours de la personne, les actions de celle-ci et les raisons qui sous-tendent ses actions. La plupart des recherches menées sur les RS optent ainsi pour une approche multiméthodologique comme : 


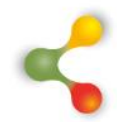

\section{REVUE HYBRIDE DE L'ÉDUCATION}

des entrevues et un questionnaire fermé (Moscovici, 1961), des entrevues et un questionnaire ouvert (Matanin et Collier, 2003), un questionnaire fermé et des focus groups (Karp et Woods, 2008), etc. Dans le cas de cette recherche, pour dégager l'influence que pouvaient avoir les RS sur le développement des pratiques évaluatives, il était nécessaire d'avoir accès à l'activité du stagiaire et aux raisons sous-jacentes à cette activité. Une méthode prisée dans la tradition ergonomique pour étudier l'activité enseignante est l'entretien d'autoconfrontation (Clot, Faïta, Fernandez et Scheller, 2000 ; Theureau, 2006) qui consiste, dans un premier temps, à filmer l'action et, dans un deuxième temps, à la visionner en compagnie de l'acteur principal. Cette méthode permet la restitution de la situation en ayant accès au contexte, aux dynamiques particulières et à l'interprétation de l'acteur. En d'autres termes, cette méthode donne accès à la construction de sens lors de l'analyse par la personne de son action dans la situation observée (Leplat, 1997 ; Teiger et Laville, 1991). En intégrant l'observation et l'entretien, il est possible de dégager les trois dimensions de l'étude des RS, à savoir le discours, les actions et les raisons de la personne.

Une fois la certification éthique de l'institution d'attache obtenue (CER. 602.184.02), la collecte de données s'est déroulée sur une période de 4 mois, soit de janvier à avril. Quatre stagiaires finissantes se sont portées volontaires pour participer aux entretiens d'autoconfrontation. Elles ont été filmées à trois reprises durant leur stage (au début, au milieu et à la fin du stage). Chaque séquence d'enseignement filmée durait entre 60 et 80 minutes et les stagiaires étaient libres de choisir la discipline et les approches pédagogiques. Après chaque séquence filmée, les stagiaires participaient à un entretien d'autoconfrontation de leur séquence d'enseignement. Chaque entretien était mené par la chercheuse et le stagiaire était seul dans la classe. Les entretiens portaient sur des actions d'évaluation en lien avec l'évaluation formative, à savoir des actions d'évaluation de l'état des connaissances des élèves (activation des connaissances antérieures), des actions de rétroactions durant la situation d'apprentissage (par les pairs, par la stagiaire ou par la situation) ainsi que des actions de régulation des apprentissages ou de l'enseignement (de l'élève ou de la stagiaire). Dans l'ensemble, les entretiens ont duré entre 65 et 105 minutes.

L'analyse des observations et des entretiens d'autoconfrontation est qualitative et se réalise selon la technique de Theureau (2006). Elle consiste à développer le cours de l'action comme le rapport qui s'instaure entre les visées d'un sujet, par rapport à une situation qu'il interprète, et ses acquis, dont émerge une représentation partielle et subjective (Van der Maren et Yvon, 2009). Concrètement, les données verbales issues de l'entretien sont confrontées à celles issues de l'observation filmée et elles sont représentées dans un tableau. La colonne de gauche comprend le verbatim et la description de la séquence observée par le stagiaire alors que la colonne de droite comprend le verbatim de l'entretien 


\section{8}

\section{REVUE HYBRIDE DE L'ÉDUCATION}

d'autoconfrontation par rapport à cette même séquence filmée. L'analyse des données se réalise selon la méthode de l'analyse thématique consistant à repérer les unités sémantiques (Negura, 2006).

\section{Résultats}

\section{L'activation des connaissances, une étape propice aux rétroactions?}

Onze séquences d'enseignement sur les 12 observées débutent par une activation des connaissances réalisée par la stagiaire. Théoriquement, l'activation des connaissances permet d'identifier les obstacles à l'apprentissage (Tardif, 1992) et de diagnostiquer les connaissances de base des élèves (De Ketele, 2010). C'est également durant cette étape que l'enseignant questionne les élèves pour leur donner des rétroactions sur leurs connaissances et permettre la régulation, soit de l'apprentissage, soit de l'enseignement (Allal et Mottier Lopez, 2007b). II s'agit donc d'une étape importante dans une séquence d'enseignement qui peut être considérée comme de l'« assessment ».

Lors des observations, les stagiaires optent pour le questionnement des élèves. Cependant, leurs rétroactions ne visent pas à s'assurer d'une bonne compréhension de l'élève ou encore de prévenir les obstacles à l'apprentissage. L'exemple du tableau 1, qui est celui d'Éva, une étudiante réalisant son stage en $3^{\mathrm{e}}$ année, illustre ce propos.

Tableau 1 : L'activation des connaissances : une étape de l'enseignement explicite

\begin{tabular}{|c|c|}
\hline Observation filmée & Propos d'Éva \\
\hline $\begin{array}{l}\text { Éva : « Cet après-midi, on va voir quelque } \\
\text { chose que tu as déjà vu en mathématique. } \\
\text { Là, il va falloir que tu retournes dans ton } \\
\text { cerveau pour les stratégies qu'on a déjà vues. } \\
\text { C'était la résolution de problème. Est-ce que } \\
\text { tu t'en souviens? } \\
\text { — Oui, disent les élèves. } \\
\text { - C'était quoi la résolution de problème? } \\
\text { demande Eva. } \\
\text { - C'était des calculs, il fallait faire des } \\
\text { additions, il fallait entourer, dit une élève. } \\
\text { - Oui, on avait des opérations à faire. Ça } \\
\text { peut être des additions, des soustractions, } \\
\text { et tu m'as parlé d'entourer, dit Éva. } \\
\text { - Ouais, il fallait entourer les chiffres, dit } \\
\text { la même élève. } \\
\text { - OK, là, tu es en train de me parler de } \\
\text { stratégies? demande Éva. Donc, la }\end{array}$ & $\begin{array}{l}\text { « Ils les } \\
\text { (stratégies) } \\
\text { connaissaient } \\
\text { déjà... C'était de } \\
\text { rafraichir les } \\
\text { connaissances. » } \\
\text { « C'était pour leur } \\
\text { montrer, tu en } \\
\text { sais déjà, tu ne } \\
\text { pars pas de } \\
\text { rien. » } \\
\text { « (Quels indices } \\
\text { repères-tu dans } \\
\text { ta séquence ?) } \\
\text { Ça me dit qu'ils } \\
\text { savent qu'il y a }\end{array}$ \\
\hline
\end{tabular}




\section{8}

REVUE HYBRIDE DE L'ÉDUCATION

\begin{tabular}{|c|c|}
\hline $\begin{array}{l}\text { résolution de problème, en fait, c'est un } \\
\text { processus qu'on fait avec les stratégies } \\
\text { pour arriver à trouver notre réponse. C'est } \\
\text { ça la résolution de problème. Tu vas en } \\
\text { faire toute ta vie, pas juste en } \\
\text { mathématique, dans toutes les autres } \\
\text { sphères de ta vie. » } \\
\text { Éva poursuit en présentant les différentes } \\
\text { stratégies et en donnant un exemple. } \\
\text { « Maintenant, je vais faire un exemple en } \\
\text { utilisant les mêmes stratégies comme si } \\
\text { j'étais vous. Moi, c'est sûr, je le fais à voix } \\
\text { haute parce que ça va te permettre de } \\
\text { comprendre où j'en suis. Si je le fais dans ma } \\
\text { tête, ça ne te permettra pas de savoir, c'est } \\
\text { quoi les stratégies que je vais faire. Je vais te } \\
\text { demander de regarder et d'écouter. C'est très } \\
\text { important parce qu'après ça, je vais te } \\
\text { demander de faire la même chose. » }\end{array}$ & $\begin{array}{l}\text { des étapes qui } \\
\text { vont aider à } \\
\text { résoudre un } \\
\text { problème. Ça } \\
\text { m'aide à savoir, } \\
\text { ah, ok, ils s'en } \\
\text { souviennent... On } \\
\text { est rendu où ? » } \\
\text { « (À propos des } \\
\text { stratégies) Pour } \\
\text { leur montrer } \\
\text { encore une fois } \\
\text { qu'ils le savaient, } \\
\text { c'était pour } \\
\text { essayer de sortir } \\
\text { les étapes à } \\
\text { faire ». }\end{array}$ \\
\hline
\end{tabular}

Selon Éva, la période d'activation des connaissances permet de voir ce que ses élèves connaissent déjà, donc de diagnostiquer où ils en sont. II y a dans son propos l'idée d'évaluer les connaissances des élèves. Or, lorsqu'on compare les actions et ses propos dans la suite de l'entretien, activer les connaissances consiste à enseigner explicitement une notion spécifique (stratégies). Elle mentionne que les élèves connaissent déjà les stratégies et, pourtant, elle s'engage très rapidement dans un enseignement explicite. Par conséquent, il semble qu'Éva ait objectivé les étapes de l'enseignement explicite telles qu'enseignées dans ses cours universitaires. Or, lors de l'ancrage, elle transforme ces étapes pour qu'elles correspondent à l'enseignement transmissif. La théorie de l'enseignement explicite insiste sur l'importance de s'appuyer sur les connaissances antérieures pour poursuivre l'enseignement (Bissonnette, Richard et Gauthier, 2006). Éva l'interprète différemment.

Le deuxième exemple présente les actions de la stagiaire Isée dans une classe de $5^{\mathrm{e}}$ année. Elle pousse le questionnement un peu plus loin qu'Éva, sans nécessairement donner des rétroactions sur la compréhension des élèves.

Tableau 2 : L'activation des connaissances : l'absence de rétroactions ciblées

\begin{tabular}{|l|l|}
\hline Observation filmée & Propos d'lsée \\
\hline
\end{tabular}




\section{8}

REVUE HYBRIDE DE L'ÉDUCATION

Isée pose la question:

«La préposition, qu'est-ce que c'est?

- C'est un mot qui introduit un verbe dans une phrase, répond Christina.

- Est-ce qu'on pourrait dire autre chose aussi ? demande Isée.

- Il y a des pronoms, il y a des noms, répond un élève.

- Donc, là, ça nous dit quelque chose. On va aller voir si d'autres personnes ont d'autres idées. Julie, François ? II y a plein d'autres choses qu'on peut dire. Tristan?

- C'est un mot invariable, mentionne

Tristan.

- Ah, un mot invariable, qu'est-ce que c'est un mot invariable? questionne Isée.

- C'est un mot qui s'écrit toujours pareil, précise un élève.

- Hum, hum, est-ce qu'il peut être au pluriel ou au singulier?

- Non.

- Non, mentionne Isée, il n'y a pas de genre, pas de nombre, il est toujours pareil comme ça. »

Isée marque une pause :

"Thomas?

- C'est un complément du groupe du verbe et du groupe du nom, précise Thomas.

- C'est quoi un complément ? demande Isée. On a quand même quelque chose d'intéressant. Est-ce que quelqu'un pourrait l'aider?

- Il y a aussi un complément de la phrase, dit François.

- Complément de la phrase aussi. Est-ce que toi François, tu peux me dire ce qu'est un complément?

- Dans une phrase, le complément de la phrase, complète la phrase, du verbe, complète le verbe, euh...

- Je pense que dans ta tête tu vois, là tu me dis pour préciser, mais tu ne sais pas exactement. Peux-tu m'en dire plus ? Christina?

- Ben, un complément c'est comme un mot qui complète la phrase, explique Christina.

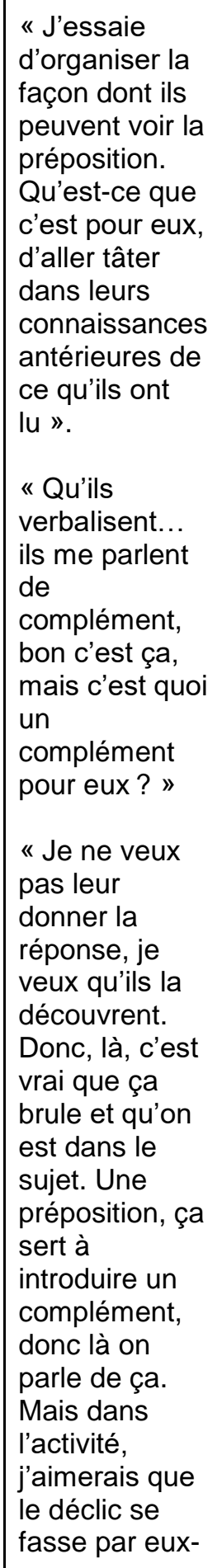




\section{8}

\section{REVUE HYBRIDE DE L'ÉDUCATION}

- Hum, hum, on parle de complément et de compléter. Oui... on va dans la direction où on veut aller. Julie?

— Ben un complément c'est comme... Un complément c'est comme à, que, pourquoi, comment, si, etc. C'est des mots invariables. - Pourquoi, tu penses ? demande Isée.

- Ben peut-être pas pourquoi, mais à, que, etc.

- On va aller voir, dit Isée. On va aller faire des petits exercices. " mêmes. Je pense que c'est plus solide quand c'est eux qui réalisent que quand c'est nous qui leur donnons tout cuit dans le bec ».

Isée questionne ses élèves pour organiser et structurer leurs connaissances. Cependant, à aucun moment elle ne valide les réponses des élèves pour s'assurer de définir la préposition. En écoutant les réponses des élèves, qui semblent deviner la réponse, la notion devient confuse pour l'ensemble de la classe. Malgré cela, elle poursuit sa situation. Après cette séquence, les élèves devaient repérer la préposition dans la phrase, ce qui s'est déroulé non sans d'importantes difficultés. En s'en allant directement à la deuxième activité, Isée ne remédie pas aux difficultés et il est très difficile pour les élèves de réguler leurs connaissances. Encore une fois, il est possible de voir un processus d'objectivation des savoirs. Isée nomme l'approche de la découverte à laquelle elle tient. Cependant, l'ancrage se réalise également dans un modèle d'enseignement transmissif.

\section{Enseigner et rétroagir comme « je l'ai vécu »}

Les stagiaires font des rétroactions aux élèves sur leur compréhension durant l'apprentissage. Or, il s'agit souvent de rétroactions données dans un enseignement direct qui visent à s'assurer que les élèves appliquent les stratégies attendues. Ce type d'enseignement est intimement lié à ce qu'ils ont connu. Voici ce que Léa nous en dit :

"D'abord, c'est celle (la méthode) que j'ai connue quand j'étais au primaire, c'est celle qu'on utilise le plus souvent, selon moi... je me suis dit que je vais faire une modélisation en avant, parce que moi je trouve ça important de leur montrer comment faire. Sûr que leur faire découvrir ça a du bon... Mais, je ne veux pas qu'ils partent avec des concepts trop erronés et qu'ils s'enlisent là-dedans. ”

Donc, si la méthode est montrée correctement au début, les élèves n'auront pas de difficulté. Léa ne semble pas prendre en considération que la notion a peut-être déjà été abordée auparavant. Les élèves ont déjà des conceptions, erronées ou non. À l'instar des propos de Léa, les analyses mettent en évidence que les stagiaires interprètent le processus de 


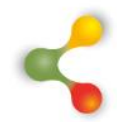

\section{REVUE HYBRIDE DE L'ÉDUCATION}

régulation comme le moment où l'élève remplace une connaissance ou une stratégie par une autre, considérée comme exacte. II n'y a donc pas de prise en compte de la complexité de l'apprentissage. L'image laissant croire que le cerveau est une page blanche qu'on remplit est encore bien ancrée chez les stagiaires. Par conséquent, les phases de rétroaction et de régulation ne peuvent apparaitre qu'en fin de séquence.

\section{L'évaluation en fin de séquence : moments de rétroaction et de régulation}

Devant l'absence d'actions et de réflexion qui mettaient en évidence des séquences de rétroactions visant à réguler les apprentissages des élèves, nous avons questionné les stagiaires sur leurs façons de faire leur permettant de s'assurer que l'élève régule ses apprentissages. En réponse à nos questions, les stagiaires ont mentionné que l'examen écrit en fin de séquence était l'action la plus déterminante pour donner des rétroactions ciblées à l'élève. C'est l'examen qui permet à l'élève de comprendre ses erreurs et d'obtenir des rétroactions.

"L'évaluation est une façon de communiquer l'information, oui aux parents, mais aussi surtout à l'enfant. Donc, justement, dans ton apprentissage, on a évalué, on a regardé comment ça allait et oui, ça va bien. On voit qu'apparemment, tu as bien compris. ॥ (propos d'lsée)

"Je pense que des notes précises, que ce soient des lettres ou des notes, c'est la même chose. Je pense que ça vient nous aider par rapport au positionnement de l'élève où il est rendu... Fait qu'avec la note, ça vient dire, petite alarme. Oh, oh, ça va pas bien, ça sonne une cloche. " (propos de Léa)

Dans les deux cas, les stagiaires identifient l'évaluation notée comme un signe de rétroaction qui mentionne à l'élève s'il est en difficulté ou non. Lorsque questionnée sur le fait de donner des rétroactions de façon descriptive, Isée répond : "Justement à l'université, les commentaires, on en parlait dans les évaluations, d'essayer d'en mettre le plus possible qu'ils soient dans le positif ou dans le négatif, toujours constructifs". Pourtant, lors des observations d'Isée, ses rétroactions arrivent toujours après une évaluation notée et déjà consignée dans le bulletin. La rétroaction perd de sa pertinence, puisque l'élève ne peut pas s'améliorer et que la note est déjà mise. Dans ce dernier exemple, celui d'Isée, il y a un modèle très clair d'objectivation et d'ancrage. La stagiaire a perçu l'importance des rétroactions écrites durant les cours universitaires, mais l'ancrage se fait dans des pratiques issues de son vécu ou de son expérience. D'ailleurs, ce propos nous permet de bien comprendre pourquoi Isée reproduit ce modèle : 


\title{
$\&$
}

\section{REVUE HYBRIDE DE L'ÉDUCATION}

\begin{abstract}
"Moi, je suis vraiment quelqu'un qui veut performer, je collectionne les $A$ et les $A+$, c'est vraiment important pour moi. On l'a vu dans le cours (à l'université), on a des motivations de performance, on veut avoir une belle note parce que ça nous valorise. Puis, il y a la motivation d'apprentissage. J'aimerais vraiment ça développer cette motivation d'apprentissage chez les élèves ».
\end{abstract}

Pour l'instant, Isée souhaiterait que la motivation de l'élève soit provoquée par d'autres éléments que ceux d'obtenir une bonne note. Cependant, en ne sachant pas comment faire, elle reproduit ce qui a fonctionné pour elle, à savoir motiver l'élève en insistant sur les notes.

Les différents propos des stagiaires mettent de l'avant que l'ancrage des savoirs théoriques, didactiques et pédagogiques se réalise selon les expériences vécues antérieurement. II n'y a pas nécessairement une remise en question sur les actions, c'est-à-dire une réflexion autour des savoirs issus des cours universitaires. Dans l'action, lorsque la situation devient difficile, les stagiaires optent pour la solution la plus viable dans leur cas. Cette solution provient souvent de leurs expériences vécues antérieurement. Ces constats nous amènent à réitérer l'importance du milieu professionnel dans l'ancrage des savoirs dans les RS et sur le développement professionnel.

\section{Perspectives dégagées des résultats : Interroger les dispositifs de formation visant l'alternance entre les milieux de stage et les milieux universitaires}

En proposant une formation en alternance, le but était de favoriser les interactions entre le discours issu de la pratique avec les discours théoriques, didactiques et pédagogiques. Les résultats de cette recherche autour de la RS de l'évaluation des apprentissages soulèvent toute la complexité de l'intégration des savoirs didactiques, pédagogiques, théoriques et expérientiels. Très rapidement, dans l'action, les stagiaires s'en remettent aux pratiques de leurs enseignants et à ce qu'elles ont vécu en tant qu'élèves. Ainsi, la démarche réflexive s'inscrit avant tout dans le contexte culturel de la profession. Ce qui se passe dans le milieu fonctionne, alors pourquoi modifier les pratiques? Cette résistance au changement, puisant dans le confort de ce qui fonctionne, demeure un défi pour la formation initiale des enseignants. Ces résultats nous amènent à proposer quelques pistes de réflexion pour la formation: repenser l'équilibre entre l'intervention du superviseur universitaire et celle de l'enseignant associé, moduler la formation en plaçant le stage comme point d'ancrage des cours universitaires et intégrer explicitement des connaissances issues de la recherche dans la formation initiale et le développement professionnel des enseignants. Le but étant que le futur enseignant, à la manière d'autres professionnels formés à l'université, 


\section{$\&$}

\section{REVUE HYBRIDE DE L'ÉDUCATION}

s'habilite à exercer un jugement professionnel en puisant des connaissances qu'il estimera appropriées à sa pratique dans la banque des savoirs pertinents pour l'éducation. II pourra ainsi éclairer et orienter son action avec plus d'assurance, ce qui est éminemment souhaitable dans la perspective d'une formation professionnalisante.

Repenser l'équilibre entre l'intervention du superviseur universitaire et celle de l'enseignant associé

Le superviseur de stage accompagne le stagiaire dans la réflexion autour des savoirs pratiques et des savoirs théoriques (Portelance, Gervais, Lessard et Beaulieu, 2008). Selon Charlier (2014), le superviseur est plutôt un expert du processus plutôt qu'un expert du contenu. Cependant, il passe beaucoup moins de temps avec le stagiaire que l'enseignant associé. Les occasions de réflexion visant à établir des liens entre les différents types de savoirs sont limitées dans le temps et la question de l'évaluation des apprentissages n'est pas nécessairement abordée. II est donc compréhensible que les stagiaires se réfèrent plus spontanément aux actions de l'enseignant associé lorsqu'il s'agit d'évaluer les apprentissages de l'élève. Par conséquent, le dispositif d'alternance n'assure pas automatiquement un arrimage entre les différents savoirs. L'accompagnement doit être repensé afin de s'assurer d'un équilibre entre les interventions de l'enseignant associé et les interventions du superviseur. À ce propos, il est nécessaire de repenser la structure de formation.

Moduler la formation en plaçant le stage comme point d'ancrage des cours universitaires

Pour l'instant, chaque année du baccalauréat en éducation préscolaire et en enseignement primaire à l'UQAC intègre une suite de cours et un stage. Des thématiques annuelles sont établies afin de s'assurer d'une cohérence entre les cours et le stage concerné, intégrant l'une ou l'autre des quatre années de formation. Cependant, il n'y a pas nécessairement de ponts planifiés entre les cours et le stage. Chaque cours vise un champ d'expertise pointu et la logique suppose que c'est l'étudiant qui, en contexte de stage, opère l'articulation d'ensemble et l'intégration des contenus des différents cours, en plus d'établir des liens entre ce contenu et les connaissances de la pratique acquises lors des stages. Or, notre recherche a mis en évidence que, pour l'évaluation des apprentissages, cette structure ne permettait pas de modifier les RS et encore moins de réinvestir des savoirs théoriques dans la pratique de l'enseignement.

Une des pistes que nous proposons est de moduler la formation en choisissant le stage comme point d'ancrage des cours. Par exemple, les cours ne seraient plus nécessairement découpés en séquence de 3 heures sur 15 semaines, tout comme les stages ne se dérouleraient pas en 


\section{6}

\section{REVUE HYBRIDE DE L'ÉDUCATION}

journées consécutives. Les cours et le stage composeraient un module spécifique qui viserait à intégrer les différents savoirs. Dans ce cas, la collaboration entre les différents intervenants, professeurs, chargés de cours, superviseurs universitaires et enseignants associés serait essentielle. Cette structure pourrait prendre la forme de séminaires d'intégration des savoirs didactiques, pédagogiques, voire praxéologiques, issus tant de la formation universitaire que de l'expérience acquise durant les stages. Par ailleurs, comme la recherche a mis en évidence l'importance de l'influence qu'exerce l'enseignant associé sur les pratiques du stagiaire, ces séminaires seraient plus riches si les enseignants associés y participaient. II y aurait là une possibilité d'ouvrir un dialogue entre les différents acteurs, de favoriser la réflexion entre les différents points de vue et de questionner les RS de chacun.

Intégrer explicitement à sa pratique des connaissances issues de la recherche en éducation

Le développement professionnel, tout comme la formation, vise une amélioration des pratiques. Des connaissances issues de la recherche suggèrent des pistes en vue d'une telle amélioration. Les séminaires, décrits dans le paragraphe précédent, pourraient être une occasion propice pour échanger autour des connaissances issues de la recherche et, pourquoi pas, démarrer de nouveaux chantiers de recherches collaboratives (Desgagné, 1997) ou de recherche-action-formation (L'Hostie, Monney et Nadeau-Tremblay, 2013). Ces deux types de recherche favorisent l'ouverture et le dialogue entre chercheurs et praticiens. Un tel dialogue est susceptible d'amener des changements dans les milieux de pratique. II y a là une option pour faire évoluer les RS, mais aussi pour poursuivre le développement professionnel de chacun des types d'acteurs concernés. En collaborant avec les enseignants associés et en se donnant des espaces de réflexion, les différents acteurs de la formation pratique se dotent de nouveaux savoirs non seulement théoriques, mais aussi issus de la pratique. Ces savoirs devraient être réinvestis dans l'accompagnement des stagiaires, mais aussi dans la recherche en éducation.

Enseigner et maitriser les gestes professionnels est une chose, mais enseigner et adopter une posture réflexive de professionnel qui prend en compte l'éclairage fourni par la recherche en éducation et qui ébranle les RS demeure un grand défi. II semble donc nécessaire de poursuivre la réflexion autour de la formation pour s'assurer que nos dispositifs amènent les futurs enseignants à développer un savoir agir cohérent avec les différentes avancées de la recherche plutôt que sur leurs propres RS. II reste maintenant à réfléchir sur la façon d'intégrer cette perspective dans un cursus universitaire de quatre ans et de faire bouger une structure administrative souvent difficilement malléable. La formation en médecine semble avoir relevé le défi, alors pourquoi pas la formation à l'enseignement? 


\section{REVUE HYBRIDE DE L'ÉDUCATION}

\section{Références}

Abric, J.-C. (1994). Pratiques sociales et représentations. Paris : Presses Universitaires de France.

Allal, L. K. et Mottier Lopez, L. (2007a). Régulation des apprentissages en situation scolaire et en formation. Bruxelles : De Boeck.

Allal, L. K. et Mottier Lopez, L. (2007b). Régulation des apprentissages : orientations conceptuelles pour la recherche et la pratique en éducation. Dans L. K. Allal et L. Mottier Lopez (dir.), Régulation des apprentissages en situation scolaire et en formation (p. 7- 23). Bruxelles : De Boeck.

Bidjang, S. G., Gauthier, C., Mellouki, M. et Desbiens, J.-F. (2005). Les finissants en enseignement sont-ils compétents? Une enquête québécoise. St-Nicolas : Les Presses de l'Université de Laval.

Bissonnette, S., Richard, M. et Gauthier, C. (2006). Comment enseigne-ton dans les écoles efficaces? Saint-Nicolas: Les Presses de L'Université de Laval.

Black, P. et Wiliam, D. (1998). Assessment and classroom learning. Assessment in Education, 5(1), 1- 54.

Bonardi, C. et Roussiau, N. (1999). Les représentations sociales. Paris : Dunod.

Buysse, A. et Vanhulle, S. (2009). Écriture réflexive et développement professionnel : quels indicateurs? Questions Vives, 5(11). Repéré à https://questionsvives.revues.org/603

Charlier, É. (2014). Accompagnement professionnel. Dans Dictionnaire des concepts de la professionnalisation (p. 17-24). Bruxelles : De Boeck.

Clot, Y., Faïta, D., Fernandez, G. et Scheller, L. (2000). Entretien en autoconfrontation croisée : une méthode en clinique de l'activité. Pistes, 2(1). Repéré à http://www.pistes.uqam.ca/v2n1/pdf/v2n1a3.pdf

de Champlain, Y. (2011). Discours sur la réussite et pratiques d'évaluation. Éducation et francophonie, 39(1), 119- 132.

De Ketele, J.-M. (2010). Ne pas se tromper d'évaluation. Revue française de linguistique appliquée, $X V, 25-37$. 


\section{REVUE HYBRIDE DE L'ÉDUCATION}

Desgagné, S. (1997). Le concept de recherche collaborative : l'idée d'un rapprochement entre chercheurs universitaires et praticiens enseignants. Revue des sciences de l'éducation, 23(2), 371- 393.

Desjardins, J. (2013). Des étudiants résistants? Mais qu'en est-il des dispositifs de formation? Dans M. Altet, J. Desjardins, R. Étienne, L. Paquay et P. Perrenoud (dir.), Former des enseignants réflexifs. Obstacles et résistances (p. 23- 38). Bruxelles : De Boeck.

Dirksen, D. J. (2013). Student Assessment: Fast, Frequent, and Formative. Lanham, MD, USA : R\&L Education. Repéré à http://site.ebrary.com/lib/uqac/docDetail.action?doclD=10805165

Doyle, M. (1997). Beyond life history as a student: Preservice teachers' beliefs about teaching and learning. College Student Journal, 31(4), 519.

Dunn, K. E. et Mulvenon, S. W. (2009). A critical review of research on formative assessment: the limited scientific evidence of the impact of formative assessment in education. Practical assessment, research and evaluation, 14(7). Repéré à http://pareonline.net/getvn.asp?v=14\&n=7

Fontaine, S., Kane, R., Duquette, O. et Savoie-Zajc, L. (2011). New teachers' career intentions: factors influencing new teachers' decisions to stay or to leave the profession. Alberta Journal of Educational Research, 57(4), 379- 408.

Hattie, J. et Timperley, H. (2007). The Power of Feedback. Review of Educational Research, 77(1), 81- 112.

Jodelet, D. (1989). Les représentations sociales. Paris : PUF.

Jorro, A. (2014). Dictionnaire des concepts de la professionnalisation. Bruxelles : De Boeck.

Karp, G. G. et Woods, M. L. (2008). Preservice Teachers' Perceptions about Assessment and Its Implementation. Journal of Teaching in Physical Education, 27(3), 327- 346.

Laurier, M. (2014). La politique québécoise d'évaluation des apprentissages et les pratiques évaluatives. Éducation et francophonie, 42(3), 31- 49.

Legendre, M.-F. (2004). Approches constructivistes et nouvelles orientations curriculaires. Dans P. Jonnaert et D. Masciotra (dir.), Constructivisme. Choix contemporains. Hommage à Ernst von 


\section{REVUE HYBRIDE DE L'ÉDUCATION}

Glasersfeld. Sainte-Foy : Presses de l'Université du Québec.

Leplat, J. (1997). Regards sur l'activité en situation de travail. Paris : PUF.

L'Hostie, M., Monney, N. et Nadeau-Tremblay, S. (2013). Une rechercheaction-formation en communauté de pratique: un projet novateur pour la formation continue des enseignants associés. Dans C. Landry et C. Garant (dir.), Formation continue, recherche et partenariat - Pour construire de nouveaux rapports entre l'université et le milieu scolaire. Ste-Foy: Presses de l'Université du Québec.

Matanin, M. et Collier, C. (2003). Longitudinal analysis of preservice teacher's beliefs about teaching physical education. Journal of Teaching in Physical Education, 22, 153- 168.

ministère de l'Éducation du Québec. (2001). La formation à l'enseignement. Les orientations. Les compétences professionnelles. Québec: Ministère de l'Éducation du Québec.

Mertler, C. A. (2009). Teachers' Assessment Knowledge and Their Perceptions of the Impact of Classroom Assessment Professional Development. Improving Schools, 12(2), 101- 113.

Monney, N. (2014). Les représentations sociales de l'évaluation des apprentissages chez des finissants d'un baccalauréat en éducation préscolaire et en enseignement primaire (BEPEP) (Doctoral dissertation, Université du Québec à Montréal).

Monney, N. et Fontaine, S. (2016). La représentation sociale de l'évaluation des apprentissages chez des finissants d'un programme universitaire en éducation préscolaire et en enseignement primaire. Mesure et évaluation en éducation, 39(2), 59-84.

Morrissette, J. (2009). Une resocialisation de l'évaluation des apprentissages. Revue canadienne des jeunes chercheures et chercheurs en éducation, 1(2), 1-8.

Moscovici, S. (1961). La psychanalyse : son image et son public. Paris : Presses universitaires de France.

Negura, L. (2006). L'analyse de contenu dans l'étude des représentations sociales. Sociologies [en ligne], Théories et recherches, mis en ligne le 22 octobre 2006. Repéré à https://journals.openedition.org/sociologies/993

Pajares, M. F. (1992). Teachers' Beliefs and Educational Research: Cleaning Up a Messy Construct. Rewiew of Educational Research, 62(3), 307- 332. 


\section{REVUE HYBRIDE DE L'ÉDUCATION}

Parker, A. et Brindley, R. (2008). Exploring Graduate Elementary Education Preservice Teachers'Initial Teaching Beliefs. The Professionnal Educator, 32(2). Repéré à https://files.eric.ed.gov/fulltext/EJ862758.pdf

Portelance, L., Gervais, C., Lessard, M. et Beaulieu, P. (2008). La formation des enseignants associés et des superviseurs universitaires. Cadre de référence. Québec. Repéré à https://www.usherbrooke.ca/education/fileadmin/sites/education/do cuments/Intranet/Documents_officiels/Rapport_Cadre_reference_2 009.pdf

Scallon, G. (1986). L'évaluation des apprentissages, le discours et la pratique. Prospectives, (Octobre), 141- 146.

Schön, D. A. (1987). Educating the reflecive practitioner: toward a new design for teaching and learning in the professions. San Francisco : Jossey-Bass.

Skamp, K. K. (2001). A longitudinal study of the influences of primary and secondary school, university and practicum on student teachers' images of effective primary science practice. International Journal of Science Education, 23(3), 227- 245.

St-Arnaud, Y. (1992). Connaître par l'action. Montréal: Presses de l'Université de Montréal.

Stiggins, R. J. (2009). Assessment FOR Learning in Upper Elementary Grades. Students learn more when they use assessments to evaluate their own learning and they have greater feelings of efficacy about their academic abilities. Phi Delta Kappan, 90(6), 419- 421.

Tardif, J. (1992). Pour un enseignement stratégique. L'apport de la psychologie cognitive. Montréal : Logiques écoles.

Teiger, C. et Laville, A. (1991). L'apprentissage de l'analyse ergonomique du travail, outil d'une formation pour l'action. Travail et emploi, 47, 53- 62.

Theureau, J. (2006). Le cours d'action. Méthode développée. Toulouse : Octares.

Uwamariya, A., \& Mukamurera, J. (2005). Le concept de «développement professionnel» en enseignement: approches théoriques. Revue des sciences de l'éducation, 31(1), 133-155.

Van der Maren, J.-M. et Yvon, F. (2009). L'analyse du travail, entre parole 


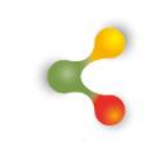

\section{REVUE HYBRIDE DE L'ÉDUCATION}

et action. Recherches Qualitatives, Hors Série(7), 42- 63.

Vanhulle, S. (2008). Au cœur de la didactique professionnelle, la subjectivation des savoirs. Dans P. Pastré et Y. Lenoir (dir.), Didactique professionnelle, didactique des savoirs professionnels. Toulouse : Octares.

Vergès, P. (1992). L'évocation de l'argent. Une méthode pour la définition du noyau central d'une représentation. Bulletin de psychologie, 45(405), 203- 209. 